enriched in evolutionary young phylostrates (bilateria, chordata, amniota and mammalia). These genes participated mostly in immunity, multicellular organism processes and differentiation. Conclusion: PLD causes de-speciation by restructuring transcription towards the upregulation of the early phylogenetic unicellular strates (cellular organisms to opistokonta) and down-regulation of the multicellularian complexity strates from (bilateralia to mammalia) indicating together with literature data concerning cancer $[1,2,3]$ that cancer-induced phylogenetic shift is associated with polyploidy component.

References: 1. Vinogradov AE. 2010. Genomics. 95:345354. 2. Trigos AS, et al. 2017. Proc Natl Acad Sci U S A.114:6406-6411. 3. Erenpreisa, et al. 2018. Cancer Hypothesis. 1: 1-20

doi: http://dx.doi.org/10.7124/bc.0009C4

\section{Pericentromeric tandem DNA transcription in malignant cells and tumour microenvironment in mice NSLC model.}

\author{
N. V. Ponomartsev, A. I. Brichkina ${ }^{2}$, \\ N. I. Enukashvily ${ }^{1 *}$ \\ ${ }^{1}$ Institute of Cytology RAS, St. Petersburg; ${ }^{2}$ Insti- \\ tute of Molecular Oncology Philipps University of \\ Marburg, Germany. \\ nie@newmail.ru
}

In higher primates, only about $1-5 \%$ of DNA is made up of protein-coding genes; the other $99 \%$ is noncoding. Most of the genome consists of repetitive DNA elements, dispersed and tandem repeats. Tandem repeats (TR, sat- ellite DNA) are located mainly in the centromere and pericentromeric regions of the chromosomes and are the DNA component of constitutive heterochromatin. In some tumours, the TR DNA decondenses and goes into a transcriptionally active state. However, it is not clear yet, what are the cells of the tumour (the stroma, malignant cells) where transcription occurs. The aim of the work was to study the transcription of TR DNA in K-rasG12D -induced carcinogenesis in mouse lung. Methods. Cancer cells and cancer-associated macrophages and fibroblasts were obtained from non-small-cell lung carcinoma (NSLC) of K-rasG12D mice. Transcription of mouse pericentromeric TR DNA (major satellite, MaSat) was analysed by qPCR, RNA-DNA FISH, immunohistochemistry methods. Results. The level of long non-coding MaSat (lncRNA) RNA in a tumour was lower than in the adjacent tissue and normal lung tissue of a healthy mouse. In the culture of NSLC epithelial tumour cells, the content of MaSat lncRNA was lower than in cancer-associated mouse fibroblasts (CAF). An increase in MaSat transcription in NSLC tumour cells occured only when apoptosis was induced by a cytotoxic drugs cisplatin, or after heat exposure. In the tumour microenvironment, the transcription was increased only in CAF but not in cancer-associated macrophages. The increase of MaSat transcription was shown both in histological specimens and in cell cultures, where CAF phenotype was induced either by co-cultivation with cancer cells or by $\mathrm{TGFb}$ treatment. The MaSat transcription was acoompanied with the appearance of markers of a CAF phenotype, including markers of cellular senescence. Transcripts were detected only in 
the nucleus, near chromocenters. No signals were detected in cytoplasm. Pre-treatment with RNAase resulted in the disappearance of signals. Conclusion. Thus, the transcription of pericentromeric satellite DNA observed in NSLC in mice occured mainly in CAF but not associated macrophages or malignant cells. Treatment with cytotoxic drugs induced appearance of CAF markers in fibroblasts. Thus, treatment with cytostatics does not induce death of CAF but promote fibroblasts transition into CAF.

The work was supported by grants from the Grants' Council of the President of RF (MK-6706.2018.7) and RSF (19-74-20102).

doi: http://dx.doi.org/10.7124/bc.0009C5

\section{Origin and evolution of nuclear localization signals}

Olga M. Lisitsyna ${ }^{1}$, Margarita A. Kurnaeva $^{2}$, Maria Y. Shubina ${ }^{1,2}$, Eugene A. Arifulin $^{1}$, Yana R. Musinova ${ }^{1,3}$, Andrey A. Mironov ${ }^{2,4,5,6}$, Eugene V. Sheval $1,7,8$

${ }^{1}$ Belozersky Institute of Physico-Chemical Biology, Lomonosov Moscow State University, Moscow, Russia; ${ }^{2}$ Faculty of Bioengineering and Bioinformatics, Lomonosov Moscow State University, Moscow, Russia; ${ }^{3}$ Koltzov Institute of Developmental Biology, Russian Academy of Sciences, Moscow, Russia; ${ }^{4}$ Skolkovo Institute of Science and Technology, Moscow, Russia; ${ }^{5}$ Institute for Information Transmission Problems, Russian Academy of Sciences, Moscow, Russia; ${ }^{6}$ Faculty of Computer Science, National Research University Higher School of Economics, Moscow, Russia;

${ }^{7}$ Faculty of Biology, Lomonosov Moscow State University, Moscow, Russia; ${ }^{8}$ LIA 1066 LFR2O
French-Russian Joint Cancer Research Laboratory, Villejuif, France.

evsheval@gmail.com

Proteins larger than $\sim 40 \mathrm{kDa}$ are selectively transfered through the nuclear pore complexes by energy-dependent mechanism that requires additional transport factors, called karyopherins, which recognize nuclear localization signals (NLSs) in their cargo proteins. Recent studies recognized some important events in the nuclear envelope evolution, and the possible ancestors of key elements of import machinery - nuclear pore complexes and karyopherins, were supposed. However, the origin and evolution of NLSs is obscure. Methods. To address this question, we analyzed the data on the NLSs and their localization relative to protein domains, and also studied possible NLS-containing regions inside prokaryotic proteins. Results. The result of the current work indicate that (1) NLS could pre-exist in prokaryotic proteins inside domains that bind nucleic acids or proteins; (2) during the course of evolution, some NLSs could be integrated within functional domains and co-evolve with them; (3) the structure of some functional domains of non-nuclear proteins allows them to function as an NLS, which requires the development of mechanisms to prevent the import of these proteins into the nucleus. Conclusions. Our data indicate that enriched with positively charged amino acids regions of nucleotide-binding domains can serve as genuine NLSs. These NLSs are integrated into domains, and their evolution might be depended on the evolution of the domain.

This work was supported by the Russian Science Foundation (grant 18-14-00195). 